\title{
ORCHESTRATION OF WEB SERVICES BASED ON TQOS USING USER AND WEB SERVICES AGENT
}

\author{
G. Tholkappia Arasu ${ }^{1}$, Ms. V.Revathi ${ }^{2}$ \\ ${ }^{1}$ Principal, AVS College of Engineering, Salem, Tamilnadu, India \\ ${ }^{2}$ Assistant Professor/IT, Adhiyamaan College of Engineering, Hosur, Tamilnadu, India
}

\begin{abstract}
A Web service is a collection of open protocols and standards for exchanging data between applications and system. Web services are platform independent and in today's internet, web services play a vital role, single service is not sufficient to respond to user's goal. To achieve the specific goal of the user, we need to combine one or more web services through the web service composition technique. Now a day's tools and environment that facilitates the composition of atomic web services into more complex web process is required. Here, to orchestrate the web service, first atomic web service among group of services is selected that must fulfil the user's requirement, transactional and Quality of Service $(Q o S)$ characteristics. Traditionally QoS based web service composition does not consider the transactional constraints during orchestration process. Therefore this paper deals with the issue of selecting and composing web services using user agent and web services agent not only according to their user requirement but also to their transactional and QoS (TQoS) properties.
\end{abstract}

Keywords: web service, TQoS, User Agent, Web services agent

\section{INTRODUCTION}

Web services are self contained application component that can be described, published located and invoked over Internet. Benefits of using web services are exposing the existing function on to network, interoperability, standardize protocol and low cost of communication. In general there are three major roles within the web service architecture:

\section{Service Provider}

This is the provider of the web service. The service provider implements the service and makes it available on the Internet.

\section{Service Requestor}

This is any consumer of the web service. The requestor utilizes an existing web service by opening a network connection and sending an XML request.

\section{Service Registry}

This is a logically centralized directory of services. The registry provides a central place where developers can publish new services or find existing ones. It therefore serves as a centralized clearinghouse for companies and their services.
$* * *$

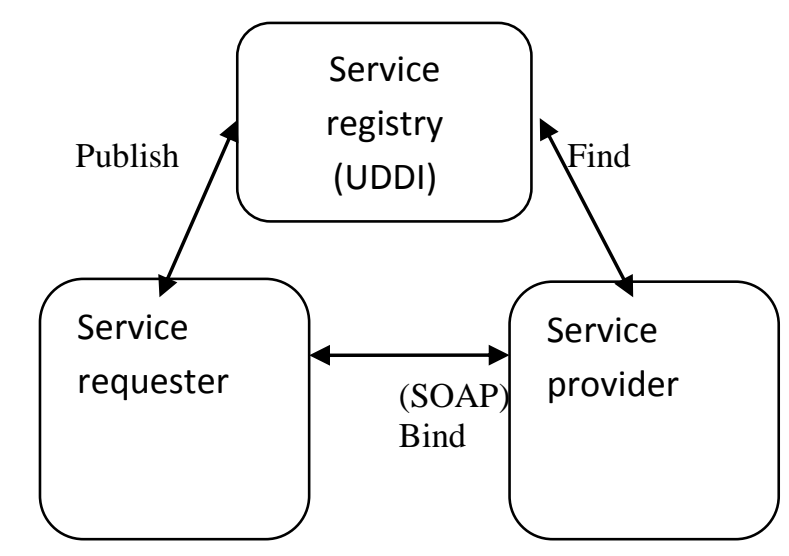

Fig -1: Web service architecture

In today's internet web service plays a vital role, single service is not sufficient to responds the user goal.

For example if a user need to book travels for holiday trip, it is not sufficient to book a train, they should take care of reserving hotels, running car, getting entertained and so on. If user executes these services one by one manually, it consumes more time and effort. For this reason, the concept of composite services is introduced to reduce the time and effort. In conventional web services composition is based on few QoS [4], [6], 7], [8], [9], [10], [11] parameters. In this paper web services composition is based on Transaction \& QoS Properties using User and Web service agents [4], [6], 7], [8], [9], [10], [11]. 
Web service composition [2], [4], [5], [7], [12], [16] is based on three step process:

1. Web service specification

2. Selection of component web service using user and web service agent based on TQoS Properties

3. Web service composition

At first step workflow [7], [13] can be used to model the web service specification. In second step selects the web service from the available web service agents, which fulfil the user's goal based on the TQoS properties using user agent. And in third step web service composition takes place. Web service agents are used to invoke the appropriate web services from available web services in web service registry. Web service agent uses SOAP(Simple Object Access Protocol) request and response messages for communication with other web services. Agent provides dynamic results. UDDI (Universal Description, Discovery, and Integration) is a standard for web services to register and make available to the consumers. Here user agent fetches the web services from the UDDI.

\section{SYSTEM ARCHITECTURE}

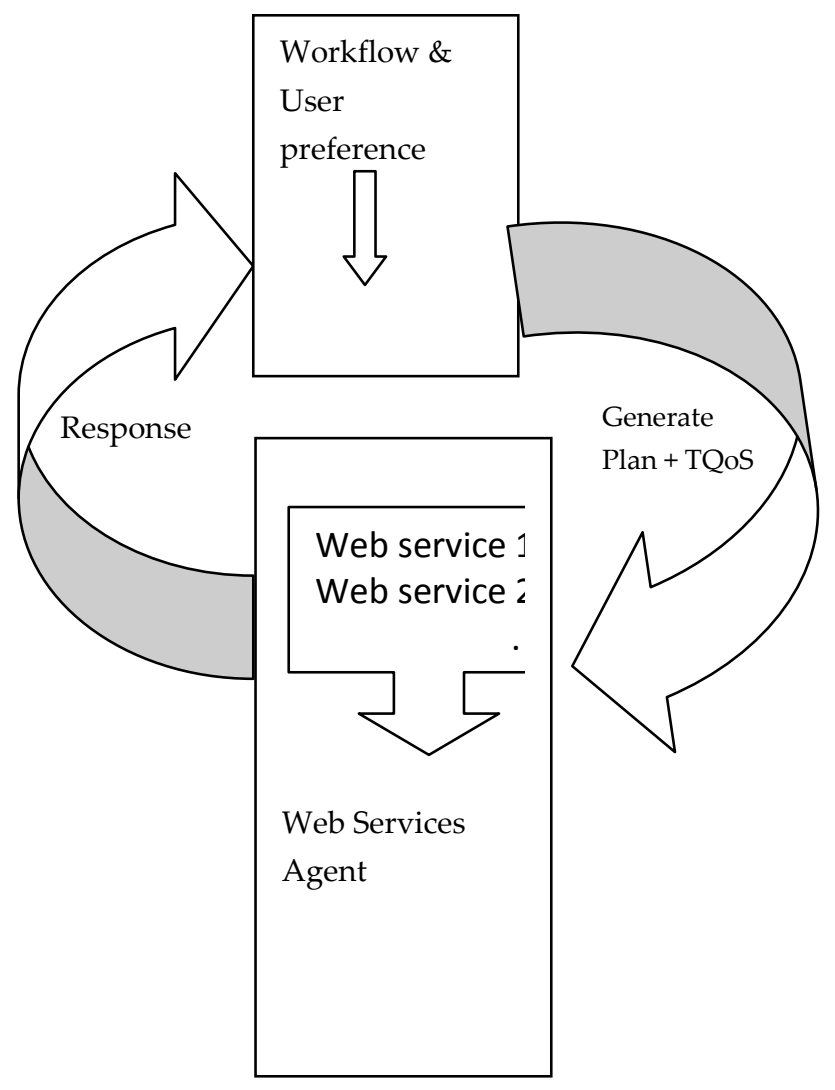

Fig -2: Overall system architecture
The overall system architecture (Fig 2) represents the Web service composition using user and web service agents. Here workflow [7], [13] defines the execution order of the activities. It denotes the basic structure of an application in terms of activities. User preferences are indicate as weight over QoS criteria. Based on the input, the user agent generates the execution plan which enforces the Transaction and QoS criteria. The web service agent will receive the execution plan as input and composite the web services from the available services according to the plan to achieve the user's goal.

\section{WEB SERVICE COMPOSITION}

Web service composition [2], [4], [5], [7], [12], [16] can be viewed as three phase. In web service specification phase, workflow of application is created by component web service. Workflow pattern can be composed by other workflows pattern [7], [13]. The following workflow patterns are used for web service composition: Sequence, parallel split (ANDsplit), exclusive choice (XOR-split), synchronization (ANDjoin) and simple merge (XOR-join).

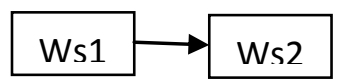

Fig - 3(a): sequential pattern

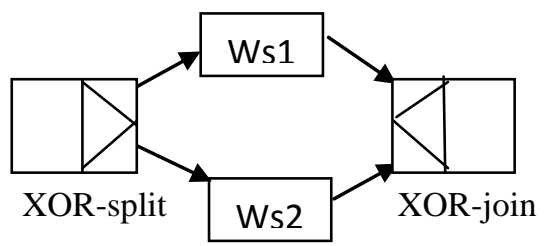

Fig -3(b): Either or workflow pattern

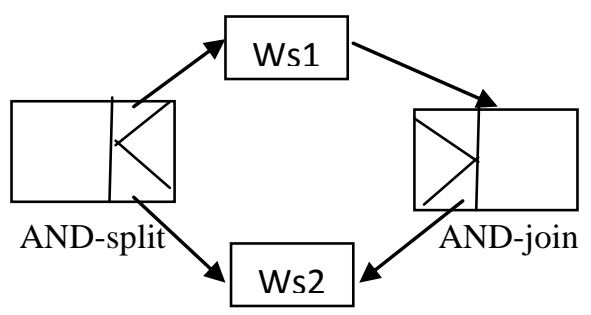

Fig -3(c): Parallel workflow pattern

User agent generates plan to selects the component web service based on the workflow pattern and user preference for service composition. In web service selection phase selects the component web service from the available web services based on the generated plan with TQoS Properties. Transactional property [1], [4], [6], [13], [14], [15] includes Pivot, compensatable and retriable web services and QoS [4], [6], 7], [8], [9], [10], [11] properties such as price, availability, 
reliability and reputation. In web service composition phase, selected web services are composite using web service agents.

Here agents are used to select and composite web services from the available atomic services. Agents [2], [3], [16], [17] are heterogeneous, autonomous and distributed system. The environment in which agents present may be static or dynamic. Agents are entities which perceive the environment by sensor and act upon that environment using actuators. User agent acts upon the user request and preference. Web service agents are used to composite the component web services based on TQoS property. For web service composition [5], [12] web service agents are used. Agents makes the composite web service reliable, robustness in response to dynamic environments.

\section{CONCLUSIONS}

In this paper, TQoS based service composition approach is used. The selection of component web service is made by Transactional properties with user's preference using user agent. Web service agents are used to provide web service composition [2], [4], [5], [7], [12], [16]. Web service composition using user agent and web service agents offer active and reliable composite web service and consistent and low cost of communication.

\section{REFERENCES}

[1]. Sami Bhiri, Olivier Perrin, Claude Godart, "Transactional Patterns for Reliable Web Services Compositions" ,In 6th international conference on Web engineering Pages 137 - 144 New York, NY, USA @2006.

[2]. Rajesh A and Rajalakshmi S , "Web Service Composition Using Multi -Agent System", In IJREAT International Journal of Research in Engineering \& Advanced Technology, Volume 1, Issue 1, March, 2013.

[3]. Müller, Ingo, and Ryszard Kowalczyk. "Service composition through agent-based coalition formation." Proc. of the first workshop on Service Composition with Semantic Web Services. 2005.

[4]. El Hadad, Joyce, Maude Manouvrier, and Marta Rukoz. "TQoS: Transactional and QoS-aware selection algorithm for automatic Web service composition." Services Computing, IEEE Transactions on 3.1 (2010): 73-85.

[5]. Q. Yu, X. Liu, B. Athman, and B. Medjahed, "Deploying and managing Web services: issues, solutions, and directions," The VLDB Journal, vol. 17, no. 3, pp. 537-572, 2008.

[6]. A. Liu, L. Huang, and Q. Li, "QoS-Aware Web Services Composition Using Transactional Composition Operator," Proc. of the7th Int. Conf. Advances in Web-Age Inf. Manag. (WAIM'2006), LNCS 4016, pp. 217-228, June 2006.

[7]. M. C. Jaeger, G. Roec-Goldmann, and G. Muehl, "QoS Aggregation for Web Service Composition using Workflow Patterns," Proc. of the 8th IEEE Int. Enterprise Distributed Object Comp. Conf. (EDOC'04), pp. 149-159, 2004.
[8]. D. Menasce, "Composing Web Services: A QoS view," IEEE Internet Computing, vol. 6, no. 8, pp. 88-90, Dec. 2004. [9]. B. Wu, CH.Chi, and S.Xu, "Service Selection Model Based on QoS Reference Vector," Proc. of theIEEE Congress on Services (Services 2007), pp. 270-277, 2007.

[10]. L. Zeng, A. N. B. Benatallah, M. Dumas, J. Kalagnanam, and H. Chang, "QoS-Aware Middleware for Web services Composition," IEEE Trans. on Software Eng., vol. 30, no. 5, pp. 311-327, May 2004.

[11]. W. Zhang, Y. Yang, S. Tang, and L. Fang, "QoS-driven Service Selection Optimization Model and Algorithms for Composite Web Services," Proc. of the 31st Annual Int. Computer Software and Applications Conf. (COMPSAC 2007), IEEE Computer Society, vol. 2, pp. 425-431, 2007.

[12]. S. Bhiri, O. Perrin, and C. Godart, "Ensuring required failure atomicity of composite Web services," Proc. of the 14th Int. Conf. on WWW, pp. 138-147, 2005.

[13]. S. Bhiri, O. Perrin, and C. Godart, "Extending workflow patterns with transactional dependencies to define reliable composite Web services," in Proc. of AICTICIW, Washington, DC, USA, 2006, p. 145.

[14]. L. Li, C. Liu, and J. Wang, "Deriving Transactional Properties of Composite Web Services," Proc. of the IEEE Int. Conf. on WebServices (ICWS 2007), pp. 631-638, July 2007. [15]. F. Montagut, R. Molva, and S. T. Golega, "Automating the Composition of Transactional Web Services," Int. J. Web Service Res., vol. 5, no. 1, pp. 24-41, 2008.

[16]. G. Vadivelu, E. Iiavarasan, and S. Prasanna, "Algorithm For Web Service Composition using Multi-Agents," in International Journal of Computer Applications, vol: 13 No.8, 2011

[17]. Shenghua Liu, Peep Kungas and Mihhail Matskin. et.al.(2006).“Agent Based Web Service Composition with JADE and JXTA“., In International Conference on Semantic Web and Web Services, 2006 